\title{
A SAS/IML program using the Kalman filter for estimating state space models
}

\author{
Fei Gu • Yiu-Fai Yung
}

Published online: 18 July 2012

(C) Psychonomic Society, Inc. 2012

\begin{abstract}
To help disseminate the knowledge and software implementation of a state space model (SSM), this article provides a SAS/IML (SAS Institute, 2010) program for estimating the parameters of general linear Gaussian SSMs using the Kalman filter algorithm. In order to use this program, the user should have SAS installed on a computer and have a valid license for SAS/IML. Since the code is completely open, it is expected that this program can be used not only by applied researchers, but also by quantitative methodologists who are interested in improving their methods and promoting SSM as a research instrument.
\end{abstract}

Keywords SAS/IML $\cdot$ Kalman filter $\cdot$ State space model

State space models (SSMs) have their origin in system theory and engineering, beginning with the groundbreaking report of Kalman (1960). As discussed by Commandeur, Koopman, and Ooms (2011), famous applications were initially (and still are) developed in astronautics for accurately tracking the

\section{F. Gu $(\bowtie)$}

Psychology and Research in Education, University of Kansas,

Lawrence, KS, USA

e-mail: fgu@ku.edu

Y.-F. Yung

SAS Institute Inc.,

Cary, NC, USA position and velocity of moving objects such as aircraft, missiles, and rockets, and were later adapted for treating time series data in econometrics (Harvey, 1989). More recently, state space methods received attention from social and behavioral scientists because of their flexibility for evaluating both the measurement properties and the concurrent and timelagged relationships among latent variables in psychological processes, by combining factor analysis and time series analysis (Chow, Ho, Hamaker, \& Dolan, 2010; Hamaker, Dolan, \& Molenaar, 2005; Ho, Shumway, \& Ombao, 2006; Molenaar \& Nesselroade, 2009; Song \& Ferrer, 2009; van Rijn, 2008; Zhang, Hamaker, \& Nesselroade, 2008). In general, state space methodology provides an effective approach for areas that can generate intensive longitudinal data (e.g., electroencephalography, functional magnetic resonance imaging, or the volatility of financial instruments). However, applications of SSM in the social sciences (except econometrics) are still uncommon.

According to Durbin and Koopman (2001), one of the few disadvantages of SSM is the lack of software packages. "In our opinion," they wrote, "the only disadvantages are the relative lack in the statistical and econometric communities of information, knowledge and software regarding these models" (p. 52). More recently, Ho, Shumway, and Ombao (2006) commented that "Though state-space modeling has become widespread over the last decade in economics and statistics, there has not been much flexible software for the statistical analysis of general models in the state-space form" (p. 159). It is worth noting that a Fortran 
program, MKFM6, developed by Dolan (2005) is freely available, and it has been used by several researchers in their studies (Chow et al., 2010; Hamaker et al., 2005; van Rijn, 2008; Zhang et al., 2008). However, Dolan noted that the source code includes a third-party function that he is not at liberty to disseminate. Due to this limitation, users will have to contact Dolan if they need to modify the program. The UCM and STATESPACE procedures for the SAS/ETS software are available for SSM, but both have their limitations. Specifically, PROC UCM can only analyze univariate time series data, while PROC STATESPACE is not capable of handling general models in state space form (i.e., the multivariate structural models discussed by Harvey, 1989, and Selukar, 2011). In addition, one big limitation of PROC STATESPACE is that the measurement model assumes a perfect measurement relationship between the state vector and the observed values by taking an identity matrix as the loading matrix. This assumption, however, is not generally true in most psychological applications. To show the parameter estimation for a general SSM, Selukar outlined an illustrative program in SAS/IML (Interactive Matrix Language) using diffuse Kalman filtering and smoothing subroutines. However, Selukar's program was written specifically for the model in his article, and thus, significant programming effort is required to adapt it for general models in state space form.

To help disseminate the knowledge and software implementation of SSM, this article provides a SAS/IML (SAS Institute, 2010) program for estimating the parameters of general linear Gaussian SSMs using the Kalman filter (KF) algorithm. In order to use this program, the user should have SAS installed on a computer and have a valid license for SAS/IML. Since the code is completely open, it is expected that this program could be used not only by applied researchers, but also by quantitative methodologists who are interested in improving their methods and in promoting SSM as a research instrument. In the following sections, this report covers (1) a brief review of the mathematical formulation of SSM, (2) a description of the KF algorithm and the KALCVF subroutine, (3) a concise introduction of the SAS/IML program, (4) an example using the program to fit an SSM to a simulated data set, (5) the results from a simulation experiment to test the program, and (6) the limitations and future development of the program.

\section{State space formulation}

The linear SSM encompasses two equations-namely, the measurement equation and the transition equation:
$y_{t}=b_{t}+H_{t} z_{t}+\varepsilon_{t}, \quad \varepsilon_{t} \sim N\left(0, \Theta_{t}\right)$

$z_{t}=a_{t}+F_{t} z_{t-1}+\eta_{t}, \quad \eta_{t} \sim N\left(0, \Psi_{t}\right)$

where $y_{t}$ is a $p \times 1$ vector of observations at time $t, b_{t}$ is a $p \times 1$ vector of intercepts at time $t, H_{t}$ is a $p \times q$ loading matrix at time $t, z_{t}$ is a $q \times 1$ vector of latent state variables at time $t, \varepsilon_{t}$ is a $p \times 1$ vector of measurement errors at time $t, a_{t}$ is a $q \times 1$ vector of constants at time $t, F_{t}$ is a $q \times q$ transition matrix at time $t$ capturing the underlying dynamic processes, and $\eta_{t}$ is a $q \times 1$ vector of transition noise at time $t$. For a Gaussian SSM, $\varepsilon_{t}$ and $\eta_{t}$ are assumed to follow a multivariate normal distribution with zero mean and covariance matrices of $\Theta_{t}$ and $\Psi_{t}$, respectively. Usually, measurement errors are assumed to be uncorrelated with each other, and thus, $\Theta_{t}$ is a diagonal matrix. The subscript $t$ indicates that all parameters $\left(a_{t}, b_{t}, F_{t}, H_{t}\right.$, $\Psi_{t}$, and $\left.\Theta_{t}\right)$ are time-varying. One recent study using the time-varying SSM is Chow, Zu, Shifren, and Zhang (2011), who presented a time-varying dynamic factor model in state space form to investigate the daily affect data over 71 days from 10 participants who had been diagnosed with Parkinson's disease. However, timevarying models are complicated and usually require extended programming skills from the researchers. In many practical applications, it is assumed that the parameters do not change over time, so that the subscript can be suppressed. The following time-invariant SSM is discussed below.

$y_{t}=b+H z_{t}+\varepsilon_{t}, \quad \varepsilon_{t} \sim N(0, \Theta)$

$z_{t}=a+F z_{t-1}+\eta_{t}, \quad \eta_{t} \sim N(0, \Psi)$

Note that the one-lag autoregressive structure in the transition equation does not mean that only a one-lag effect can be incorporated. Rather, a lag effect of arbitrary order can be put into state space form. A bivariate series $\left(y_{t 1}, y_{t 2}\right)$ secondorder vector autoregressive and first-order moving average $[\operatorname{VARMA}(2,1)]$ process is

$$
\begin{aligned}
\left(\begin{array}{l}
y_{t 1} \\
y_{t 2}
\end{array}\right)= & \left(\begin{array}{ll}
\varphi_{1} & \varphi_{2} \\
\varphi_{3} & \varphi_{4}
\end{array}\right)\left(\begin{array}{l}
y_{t-1,1} \\
y_{t-1,2}
\end{array}\right)+\left(\begin{array}{ll}
\varphi_{5} & \varphi_{6} \\
\varphi_{7} & \varphi_{8}
\end{array}\right)\left(\begin{array}{l}
y_{t-2,1} \\
y_{t-2,2}
\end{array}\right) \\
& +\left(\begin{array}{l}
u_{t 1} \\
u_{t 2}
\end{array}\right)+\left(\begin{array}{cc}
\theta_{1} & 0 \\
0 & \theta_{2}
\end{array}\right)\left(\begin{array}{l}
u_{t-1,1} \\
u_{t-1,2}
\end{array}\right),
\end{aligned}
$$

$t=0, \pm 1, \pm 2, \ldots$ 
The state space representation of this process is obtained by defining

$$
\begin{aligned}
& y_{t}=\left(\begin{array}{c}
y_{t 1} \\
y_{t 2}
\end{array}\right), z_{t}=\left(\begin{array}{c}
y_{t 1} \\
y_{t 2} \\
y_{t-11} \\
y_{t-1,2} \\
u_{t 1} \\
u_{t 2}
\end{array}\right), z_{t-1}=\left(\begin{array}{c}
y_{t-11} \\
y_{t-1,2} \\
y_{t-2,1} \\
y_{t-2,2} \\
u_{t-1,1} \\
u_{t-1,2}
\end{array}\right) \\
& b=\left(\begin{array}{l}
0 \\
0
\end{array}\right), H=\left(\begin{array}{llllll}
1 & 0 & 0 & 0 & 0 & 0 \\
0 & 1 & 0 & 0 & 0 & 0
\end{array}\right), \Theta=\left(\begin{array}{ll}
0 & 0 \\
0 & 0
\end{array}\right) \\
& a=\left(\begin{array}{l}
0 \\
0 \\
0 \\
0 \\
0 \\
0
\end{array}\right), F=\left(\begin{array}{cccccc}
\varphi_{1} & \varphi_{2} & \varphi_{5} & \varphi_{6} & \theta_{1} & 0 \\
\varphi_{3} & \varphi_{4} & \varphi_{7} & \varphi_{8} & 0 & \theta_{2} \\
1 & 0 & 0 & 0 & 0 & 0 \\
0 & 1 & 0 & 0 & 0 & 0 \\
0 & 0 & 0 & 0 & 0 & 0 \\
0 & 0 & 0 & 0 & 0 & 0
\end{array}\right), \eta_{t}=\left(\begin{array}{c}
u_{t 1} \\
u_{t 2} \\
0 \\
0 \\
u_{t 1} \\
u_{t 2}
\end{array}\right), \Psi=\left(\begin{array}{cccccc}
\psi_{1} & 0 & 0 & 0 & 0 & 0 \\
0 & \psi_{2} & 0 & 0 & 0 & 0 \\
0 & 0 & 0 & 0 & 0 & 0 \\
0 & 0 & 0 & 0 & 0 & 0 \\
0 & 0 & 0 & 0 & 0 & 0 \\
0 & 0 & 0 & 0 & 0 & 0
\end{array}\right)
\end{aligned}
$$

The state space representation of a general $\operatorname{VARMA}(p, q)$ process is given by Aoki (1987), Hannan and Deistler (1988), Lütkepohl (2005), and Wei (1990).

\section{The KF algorithm and the KALCVF subroutine}

The KF algorithm is used to provide the normal theory/ Gaussian maximum-likelihood (ML) estimates via any optimization technique. Beginning with the initial state variables $\left(z_{1 \mid 0}\right)$ and the associated covariance matrix $\left(P_{1 \mid 0}\right)$, the $\mathrm{KF}$ algorithm uses two steps (i.e., the prediction and filtering steps). In the prediction step, the conditional expectation of state variables and the corresponding covariance matrix are estimated at the current observation using all prior observations; then, these estimates are updated using the actual current observation in the filtering step. Specifically, the prediction step is initialized at the first observation $(t=1)$ using the initial values

$z_{1 \mid 0}=a+F z_{0 \mid 0}$,

$P_{1 \mid 0}=F P_{0 \mid 0} F^{\prime}+\Psi$

The one-step-ahead prediction error and its associated covariance matrix are computed as

$e_{1}=y_{1}-y_{1 \mid 0}=y_{1}-\left(b+H z_{1 \mid 0}\right)$,

$D_{1}=H P_{1 \mid 0} H^{\prime}+\Theta$, and then these estimates are updated in the filtering step

$z_{1 \mid 1}=z_{1 \mid 0}+P_{1 \mid 0} H^{\prime} D_{1}^{-1} e_{1}$,

$P_{1 \mid 1}=P_{1 \mid 0}-P_{1 \mid 0} H^{\prime} D_{1}^{-1} H P_{1 \mid 0}$.

Taking the values of $z_{1 \mid 1}$ and $P_{1 \mid 1}$, the prediction step and the filtering step are recursively implemented at the second observation, and so on. For $t=1,2, \ldots, T$, the Kalman recursion can be written as

$z_{t \mid t-1}=a+F z_{t-1 \mid t-1}$

$P_{t \mid t-1}=F P_{t-1 \mid t-1} F^{\prime}+\Psi$

$e_{t}=y_{t}-y_{t \mid t-1}=y_{t}-\left(b+H z_{t \mid t-1}\right)$

$D_{t}=H P_{t \mid t-1} H^{\prime}+\Theta$

$K_{t}=P_{t \mid t-1} H^{\prime} D_{t}^{-1}$

$z_{t \mid t}=z_{t \mid t-1}+K_{t} e_{t}=z_{t \mid t-1}+P_{t \mid t-1} H^{\prime} D_{t}^{-1} e_{t}$

$P_{t \mid t}=\left(I-K_{t} H\right) P_{t \mid t-1}=P_{t \mid t-1}-P_{t \mid t-1} H^{\prime} D_{t}^{-1} H P_{t \mid t-1}$, 
where $K$ is called the Kalman gain matrix. After the KF cycles through all observations, $e_{t}$ and $D_{t}(t=1,2, \ldots, T)$ are readily available to be substituted into the log-likelihood function based on the assumption of a multivariate normal distribution given by Schweppe (1965), and this function is referred to as the prediction error decomposition (PED):

$\mathrm{PED}=\frac{1}{2} \sum_{t=1}^{T}\left[-p \log (2 \pi)-\log \left|D_{t}\right|-e_{t}^{\prime} D_{t}^{-1} e_{t}\right]$.
Finally, ML estimates can be obtained by maximizing PED with respect to the parameters $(a, b, F, H, \Psi$, and $\Theta)$.

Given known values of $a, b, F, H, \Psi$, and $\Theta$, the KALCVF subroutine in SAS/IML can implement the KF algorithm just described, ${ }^{1}$ and it can also compute $k$-stepahead estimates, a process that is not discussed in this article. To call the KALCVF subroutine, the user needs to submit the following syntax in an IML session:

CALL KALCVF (pred, vpred, filt, vfilt, data, lead, a, f, b, h, var, z0, vz0);

The required input arguments include data, lead, $\mathrm{a}, \mathrm{f}, \mathrm{b}, \mathrm{h}$, and var and the optional input arguments are z 0 and $v z 0$. The data argument is a matrix of observed series, $\left[y_{1}, y_{2}, \ldots, y_{T}\right]^{\prime}$. The lead argument is the number of steps to forecast after the end of the data, and it is always set to 0 because the purpose is constrained to estimate parameters, and no future prediction is needed for this purpose. Initialstate variables $\left(z_{1 \mid 0}\right)$ and the corresponding covariance matrix $\left(P_{1 \mid 0}\right)$ are specified by z 0 and $\mathrm{vz} 0$ respectively. The arguments $a, f, b$, and $h$ correspond according to the parameters in the State Space Formulation section, whereas var is the block diagonal matrix made up of $\Psi$ and $\Theta$. That is, var $=$ $\operatorname{diag}[\Psi \Theta]$.

Naturally, the var matrix is nonnegative definite, since both $\Psi$ and $\Theta$ are covariance matrices. However, this implicit requirement may be violated when using the KALCVF subroutine during the optimization process, which will in turn stop the optimization from searching the ML estimates. In the following example, the solution to avoid this numeric problem is to place boundary constraints on certain elements in the parameters. Depending on the model identification method, other solutions are also possible. This issue will be discussed in the subsequent illustrative example.

The returned arguments of the KALCVF subroutine are pred, vpred, filt, and vfilt. The pred argument contains all estimates stacked vertically from the prediction step-that is, $\left[z_{1 \mid 0}, z_{2 \mid 1}, \ldots, z_{T \mid T-1}, z_{T+1 \mid T}, \ldots, z_{T+\text { lead } \mid T]^{\prime}-}\right.$ and the vpred argument contains all of the associated covariance matrices stacked vertically - that is, $\left[P_{1 \mid 0}, P_{2 \mid 1}, \ldots\right.$, $\left.P_{T \mid T-1}, P_{T+1 \mid T}, \ldots, P_{T+\text { lead } \mid T}\right]^{\prime}$. Finally, the filt and vfilt arguments are $\left[z_{1 \mid 1}, z_{2 \mid 2}, \ldots, z_{T \mid T}\right]^{\prime}$ and $\left[P_{1 \mid 1}, P_{2 \mid 2}, \ldots\right.$, $\left.P_{T \mid T}\right]^{\prime}$, separately.

Whenever a missing value is encountered in the matrix of observed series, the KALCVF subroutine uses the conditional expectation from the previous time point to substitute for the missing value and treats it as the observed value to proceed to the next time point.

\section{A SAS/IML program for fitting SSM}

A SAS/IML program was written to estimate the parameters of SSM. In this program, the KF algorithm is implemented in the KALCVF subroutine to compute the negative PED, which converts the maximization problem to the minimization problem, and the NLPQN (standing for "nonlinear programming quasi-Newton") subroutine is used to accomplish the optimization. According to the SAS documentation, the NLPQN subroutine computes an optimum value of a continuous function by using a quasi-Newton method.

The program in Listing 1 contains four parts. In the first part, three modules are defined (i.e., op_fun, stack_parm, and unpack_parm). Given known values of the parameters $(a, b$, $F, H, \Psi$, and $\Theta$ ), the op_fun module computes a value from the negative PED using the KF algorithm; the stack_parm module produces a row vector (parm) by extracting all unique elements from every parameter matrix; and the unpack_parm module reverses the stack_parm module - that is, elements in the parm vector are transformed back into their original matrix form.

In the second part, model specification information is collected. To specify a model, the user needs to tell the program whether a particular parameter matrix is fixed or freely estimated. Also, a set of values are needed for all unique elements of the parameter matrices. Those values will be provided in matrix form through some SAS macro variables. Depending on the model specification, the program will print the initial estimates for those parameter matrices to be freely estimated.

In the third part, the NLPQN subroutine will use the starting values provided by the user and run the three

\footnotetext{
${ }^{1}$ The KALCVF subroutine can also implement the KF algorithm with known time-varying parameters $\left(a_{t}, b_{t}, F_{t}, H_{t}, \Psi_{t}\right.$, and $\left.\Theta_{t}\right)$. Readers should refer to the SAS/IML 9.22 User's Guide (SAS Institute, 2010, pp. 758-761) for technical details.
} 
Listing 1 A SAS/IML program to estimate parameters of linear state space model

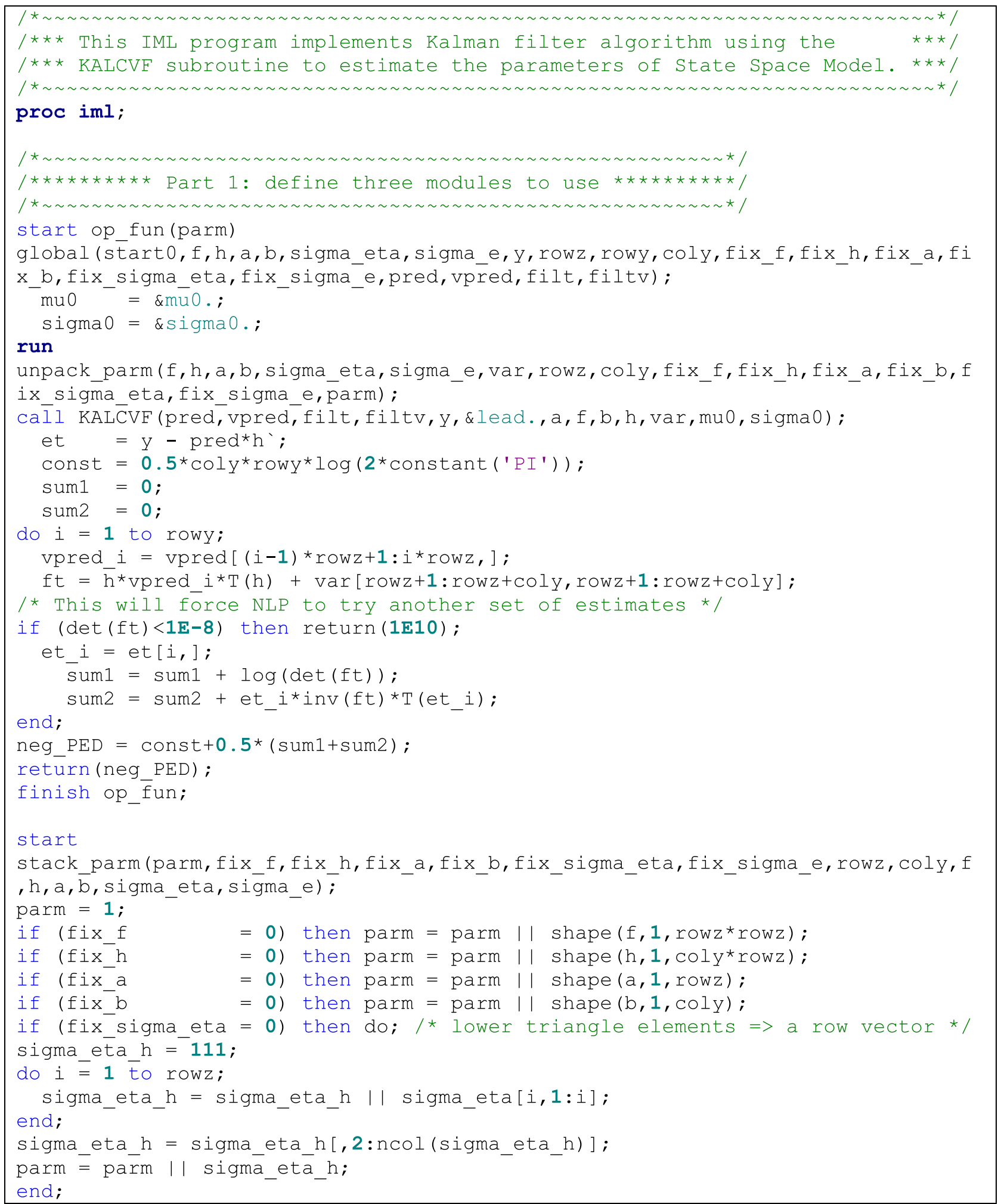




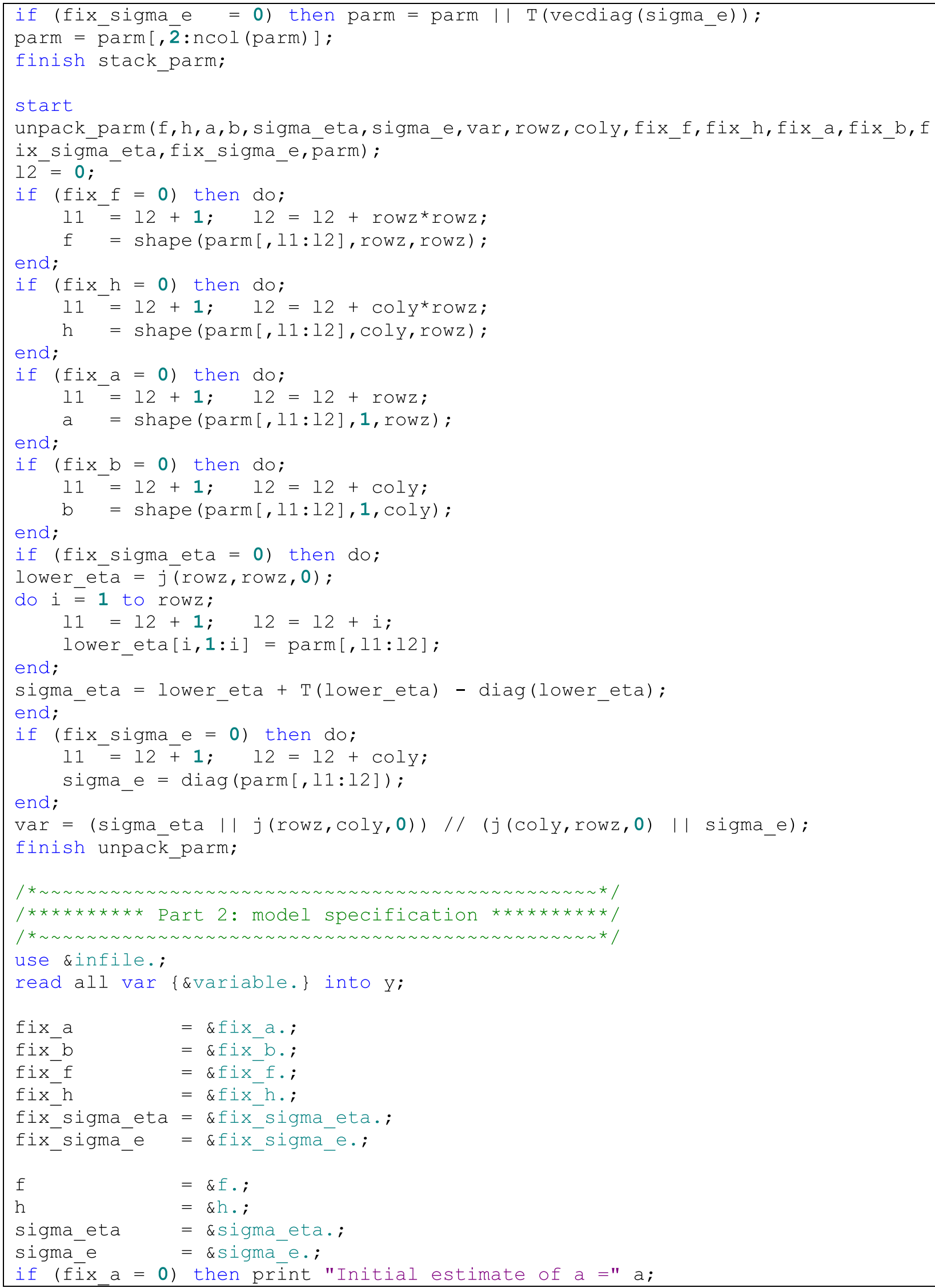




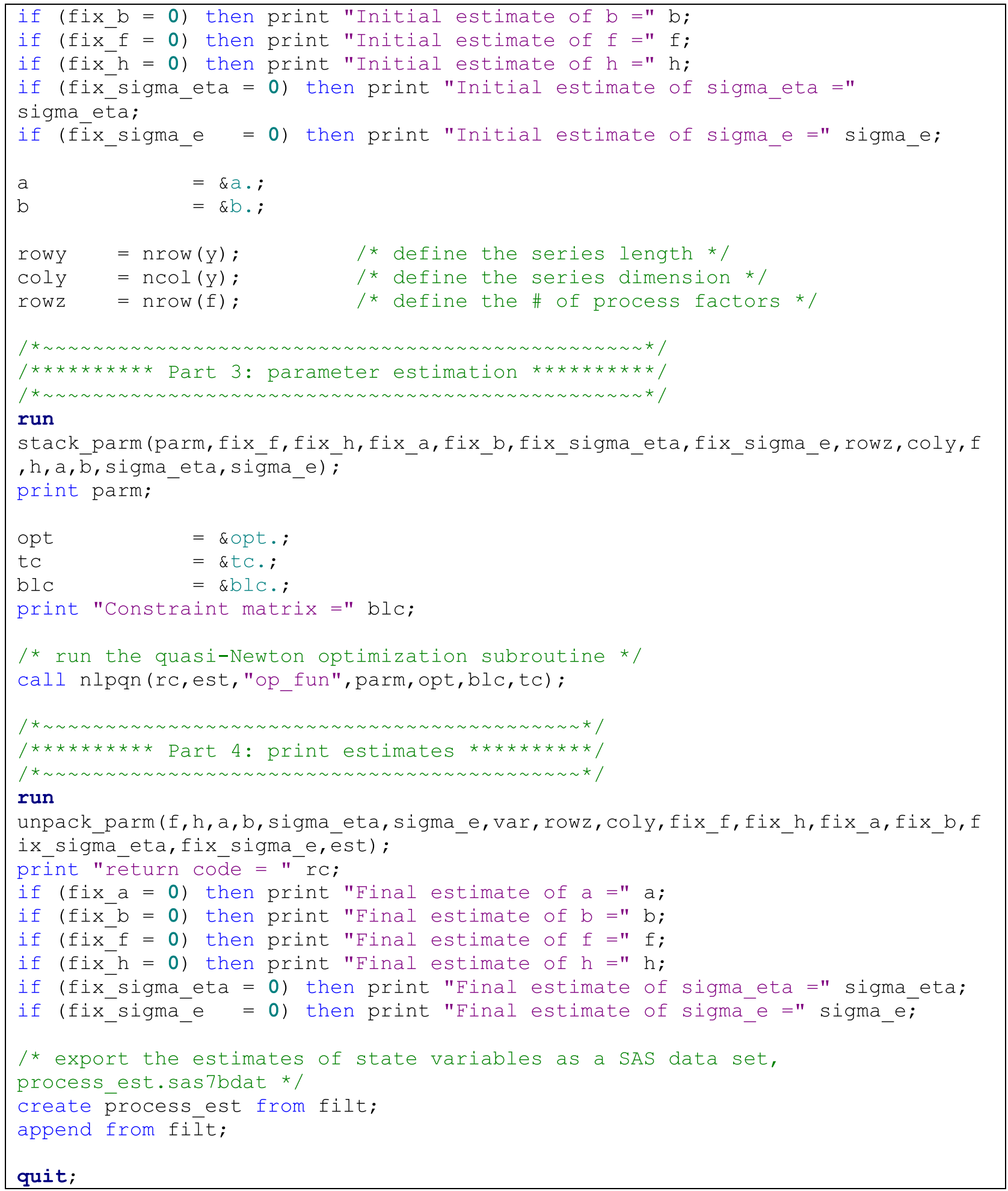

modules iteratively to find the ML estimates. Three additional matrices are required as options for the NLPQN subroutine. These options inform the NLPQN subroutine whether it is a minimization or maximization problem, the termination criteria, and the boundary constraints placed.
Technical details of the options for the optimization subroutines in SAS/IML can be found from the SAS/IML 9.22 User's Guide (chap. 14, SAS Institute, 2010).

In the last part, the program prints the final estimates in their original matrix forms. The IML program is saved as 
KF. sas in the same folder as the other program, as is illustrated in the next section.

\section{Fitting SSM to a simulated data set: An example}

To illustrate the SAS/IML program for parameter estimation, a time series data set with 500 observations was generated from a dynamic factor model provided by Zhang et al. (2008), and the same data generation procedures were followed that had been used by Zhang et al. (pp. 390-391). This example is used because, in psychological applications, modeling human dynamics using latent factors is more appropriate than using the observed variables in the conventional VARMA approach. In addition, the measurement properties can be evaluated in the measurement equation. In other words, the loading matrix (i.e., $H$ ) can be freely estimated in the model. Specifically, the model includes six observed variables indicating two factors, and each of the factors is indicated by three variables separately. Within the same time point, the two factors are correlated, while be- tween two consecutive time points, the factors have an autoregressive and cross-regressive effect.

Figure 1 portrays this dynamic factor model, in which the intercepts, $b$, and the constants, $a$, are both fixed to zero. For parameter matrices to be freely estimated, each unique element is labeled as $X_{j}$, and their true values are also provided in matrix notation. The reason of labeling parameters using $X_{j}$ instead of the conventional notation for different model components (e.g., $\lambda$, $\psi, \beta$, or $\theta)$ is that the ordered subscript, $j$, provides a convenient way to index the columns of the row vector created by the stack_parm module, and those columns in turn correspond to the columns in the boundary constraint matrix. It is crucial that the boundary constraints be correctly specified. This point will be illustrated with the discussion of fixed or free parameters in this example.

In total, the model contains 25 unique elements, and some of these elements are fixed. For example, $X_{17}$ and $X_{19}$ are fixed to 0.36 for model identification, whereas $X_{6}$, $X_{8}, X_{10}, X_{11}, X_{13}$, and $X_{15}$ are fixed to 0 for specification reasons:

$$
\begin{aligned}
& F=\left[\begin{array}{ll}
X_{1} & X_{2} \\
X_{3} & X_{4}
\end{array}\right]=\left[\begin{array}{cc}
.8 & 0 \\
0 & .8
\end{array}\right] \quad \Psi=\left[\begin{array}{ll}
X_{17}^{*} & X_{18} \\
X_{18} & X_{19}^{*}
\end{array}\right]=\left[\begin{array}{ll}
.36 & .18 \\
.18 & .36
\end{array}\right]
\end{aligned}
$$

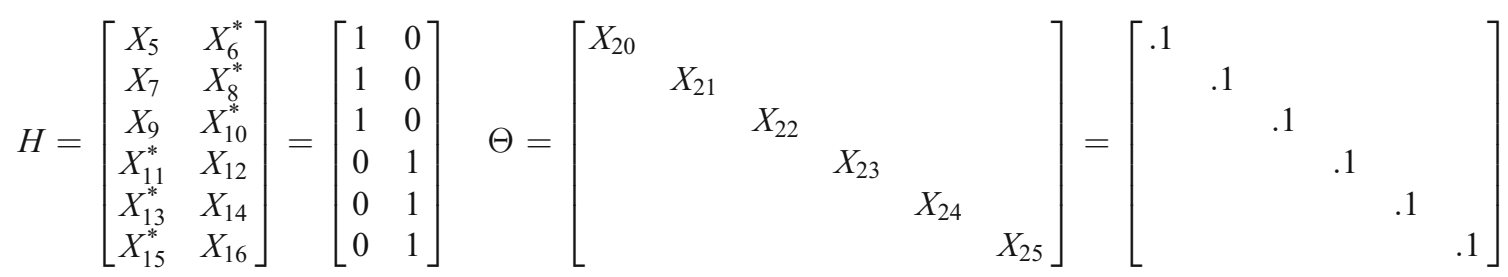

More details about the fixed or free parameters in the NLPQN subroutine will be discussed later.

As was pointed out by one anonymous reviewer, stationarity is an important concept in time series analysis. The state vector in this example is stationary because all roots of the determinantal equation

$|I-\lambda F|=0$

lie outside the complex unit circle. A thorough discussion of stationarity in the context of multivariate time series is beyond the scope of this article, but readers can refer to Lütkepohl (2005) for details.

The program in Listing 2 has two IML sessions. The first IML session generates a SAS data set, observed, in which there are six observed variables, $y 1-y 6$. Then, a set of $\%$ LET statements assign the required information to SAS macro variables, which will be used to set up a model. Specifically, \% LET INFILE $=$ and \% LET VARIABLE = tell SAS the data set and the variables to be analyzed. \% LET $\mathrm{LEAD}=0$, since no forecasting is made. A mean vector and covariance matrix provided by $\%$ LET MU0 $=$ and $\%$ LET SIGMA0 $=$ will be used to initialize the KF algorithm. It is worth noting that the $\%$ STR function is used to input the necessary information in proper matrix form. Next, a \% LET FIX_MATRIX (MATRIX can represent $a_{t}, b_{t}, F_{t}, H_{t}, \Psi_{t}$, and $\Theta_{t}$, separately) statement is used to fix or free a matrix in the model. If \% LET FIX MATRIX $=1$, the matrix is fixed, and if \% LET FIX MATRIX $=0$, the matrix is freely estimated. No matter whether a matrix is fixed or freely estimated, numeric values are needed for each matrix, and these 


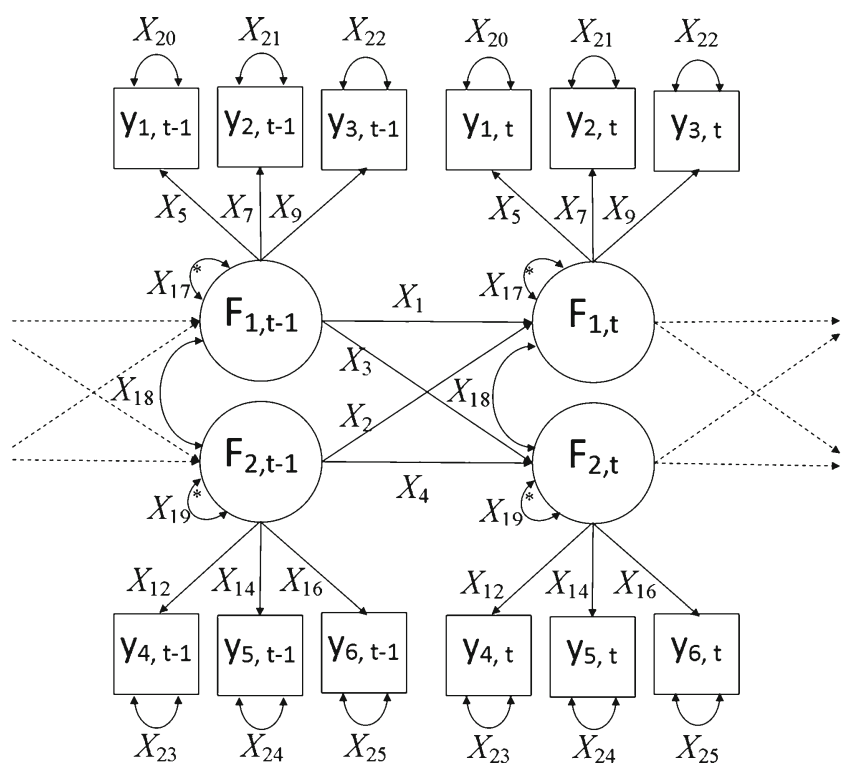

Fig. 1 The simulated model with six observed variables and two factors. The factor scores have a one-lag autoregressive structure. ${ }^{*} X_{17}$ and $X_{19}$ are fixed to 0.36 for model identification

values are treated as fixed values if the matrix is fixed, or starting values if the matrix is freely estimated. Supplying the values to each matrix is done by a $\%$ LET MATRIX = statement in matrix form in the \% STR function. Since the purpose of this example is to illustrate the parameter estimation, true values are used as starting values for matrices that are freely estimated. The last three \% LET statements provide the NLPQN subroutine some computational options. The values specified in \% LET OPT $=$ and \% LET TC $=$ can be used at their default settings in most applications with success. However, \% LET BLC $=$ assigns the options for the NLPQN subroutine to establish boundary constraints, which does need particular attention. The use of this option will be described shortly after this paragraph, as the solution in the illustrative example to avoid negative definiteness of the var matrix. Finally, the last line of code (i.e., the \% INCLUDE statement) runs the SAS/IML program in a separate IML session to fit the model. In short, model specification is similar to that in most commercial software packages.

After the stack parm module produces the row vector, parm $=\left[X_{1} X_{2} \ldots X_{25}\right]$, using all unique elements from the parameter matrices to be freely estimated, the user can define a corresponding matrix ${ }^{2}$ assigned to the $\mathrm{blc}$ macro variable to place boundary constraints on these elements. In this example, the blc matrix has two rows and 25 columns. Each column in the blc matrix corresponds to an element in the parm vector. Within a column, the first row is used to set up the lower bound,

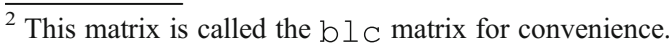

and the second row is used to place the upper bound. A dot in the blc matrix indicates that no boundary constraint is placed. It can be shown that ensuring a nonnegative definite $\Psi$ matrix is a sufficient condition $^{3}$ to meet the requirement of nonnegative definiteness of the var matrix used in the KALCVF subroutine, and this condition is used as the solution to avoid numeric difficulty in this example.

According to the model specification, both rows for the 6th, 8th, 10th, 11th, 13th, and 15th columns are set to 0 in order to fix these factor loadings (that is, $X_{6}=$ $X_{8}=X_{10}=X_{11}=X_{13}=X_{15}=0$ ). In addition, both rows for the 17 th and 19th columns are set to 0.36 to fix the diagonal elements in the $\Psi$ matrix for model identification (i.e., $X_{17}=X_{19}=0.36$ ). In general, an element can be fixed by setting the same value on the lower and upper bounds. Furthermore, the values on the first row for the 20th through 25 th columns are set to 0 to ensure nonnegative variances of the measurement error in the $\Theta$ matrix (that is, $X_{20}$ through $X_{25}$ are greater than or equal to 0 ). Finally, the 18 th column will be used to ensure the nonnegative definiteness of the var matrix.

In this example, $\Psi$ is a $2 \times 2$ matrix, and nonnegative definiteness indicates that

$X_{17} X_{19}-X_{18}^{2} \geq 0$.

Since $X_{17}$ and $X_{19}$ are both fixed to 0.36 , this requirement is equivalent to placing two boundary constraints on $X_{18}$. That is,

$X_{18} \geq-0.36$,

$X_{18} \leq 0.36$.

Therefore, the first row of the 18 th column is set to -0.36 , and the second row to 0.36 . Other possible solutions include placing linear and/or nonlinear constraints, which may or may not be easy to implement. Or, the user can even modify the op fun module in certain cases. For example, if the model is identified by fixing one of the loadings on each state variable in the $H$ matrix (also known as the "marker variable method," in the structural equation modeling parlance) while leaving all elements in the $\Psi$ matrix to be freely estimated, the user can add an IF statement to force the $\mathrm{op}$ _ fun module to return a very large value (e.g., 1E10) in case the determinant of the var matrix is really small (e.g., $1 \mathrm{E}-8)$. As a result, the

\footnotetext{
$\overline{3}$ By definition, given $\operatorname{var}=\operatorname{diag}[\Psi \Theta]$ and $\forall$ non-null vector $\theta=\left[\theta_{1}\right.$ $\left.\theta_{2}\right]^{\prime}$, if $\theta^{`} \operatorname{var} \theta=\theta_{1}{ }^{\prime} \Psi \theta_{1}+\theta_{2}{ }^{\prime} \Theta \theta_{2} \geq 0$, then $\operatorname{var}$ is nonnegative definite. Assuming that at least one (or both) of the $\theta_{1}$ and $\theta_{2}$ vectors is non-null, if $\theta_{2}{ }^{`} \Theta \theta_{2} \geq 0$ is already known, a sufficient condition to ensure the nonnegativeness of $\operatorname{var}$ is $\theta_{1}{ }^{\prime} \Psi \theta_{1} \geq 0$.
} 
Listing 2 An example program

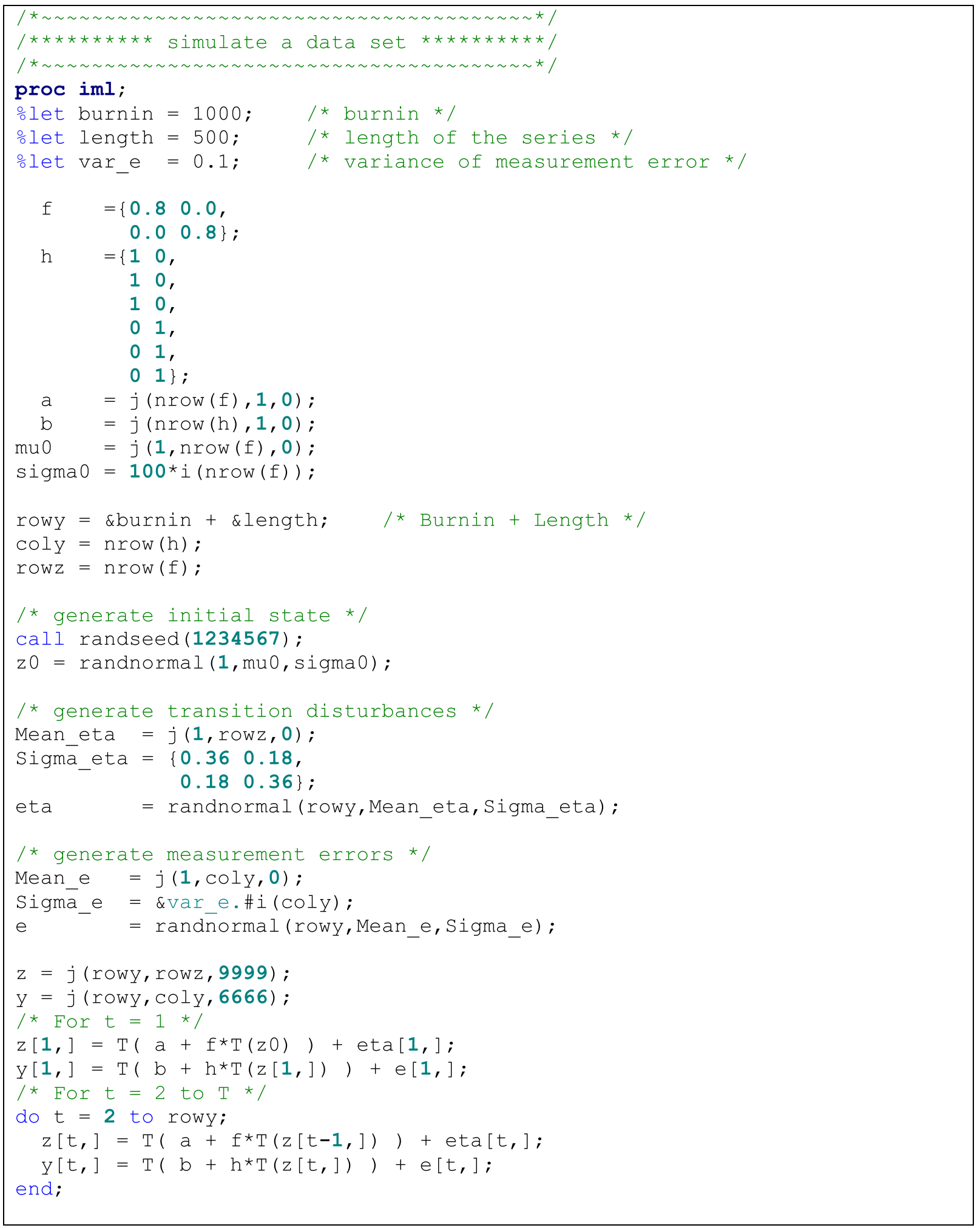

NLPQN subroutine will have to try another set of estimates before using the var matrix in the KALCVF subroutine. This approach can be easily implemented by inserting the following IF statement before the KALCVF statement in the op_fun module:

if $(\operatorname{det}(\operatorname{var})<1 \mathrm{E}-8)$ then return $(1 \mathrm{E} 10)$; 


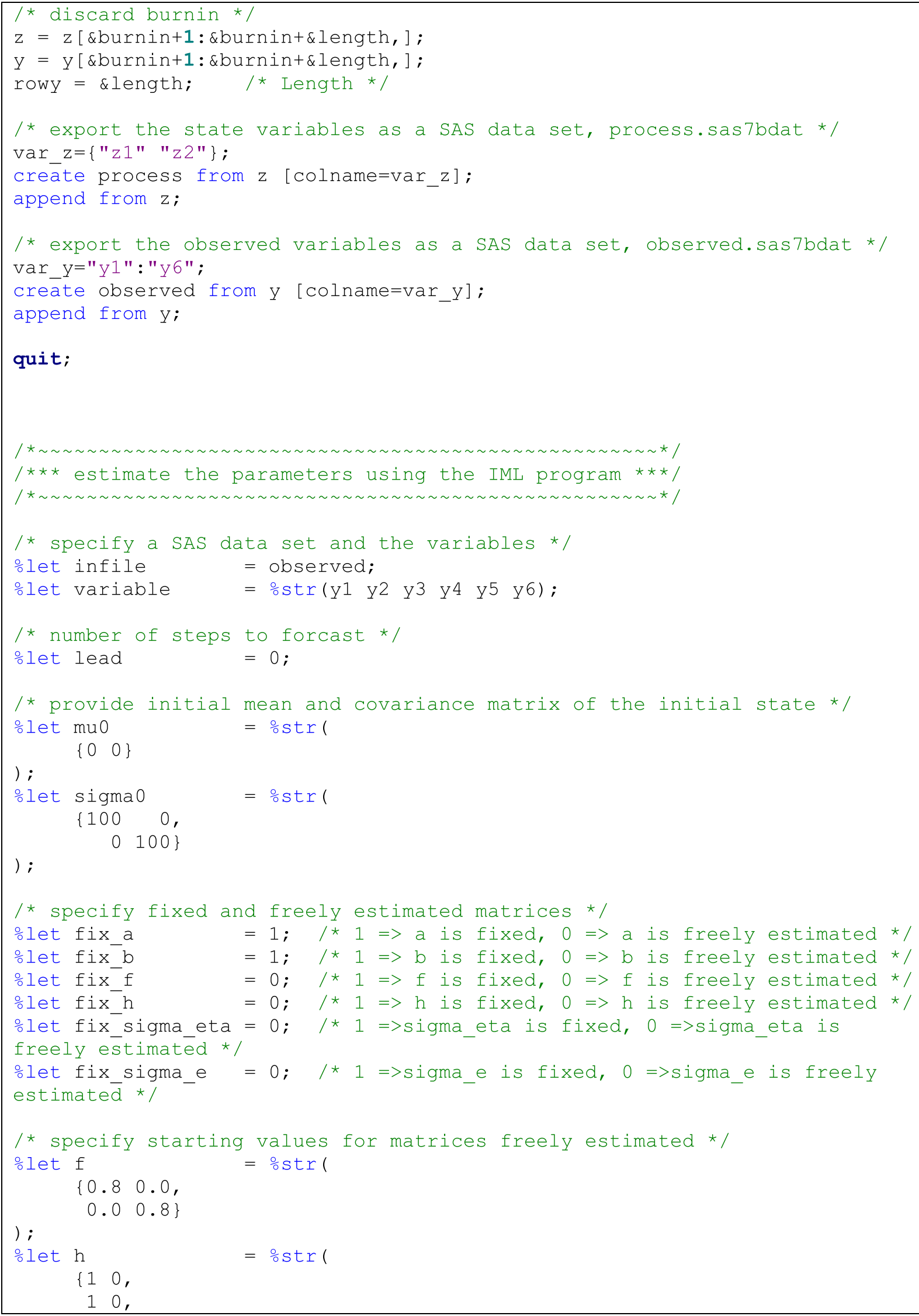




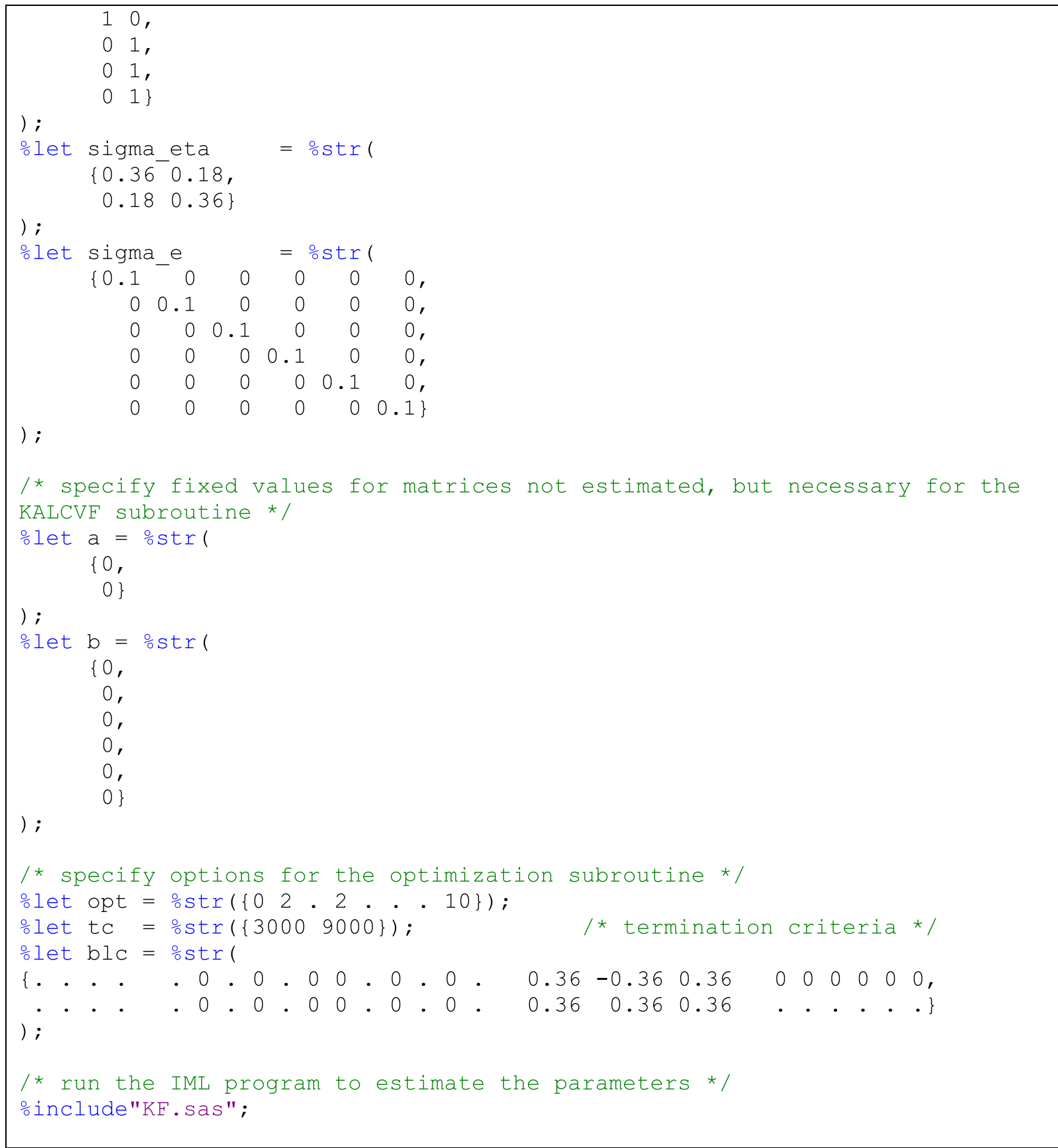

Corresponding to this model identification method, the blc matrix will be assigned as such in Listing 2:

$$
\begin{aligned}
& \text { 이 } \mathrm{blc}=\frac{\mathrm{o}}{\mathrm{str}}(
\end{aligned}
$$

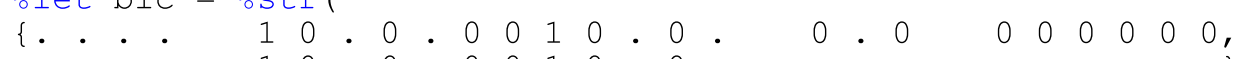

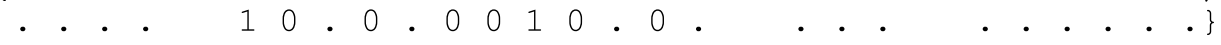

$$
\begin{aligned}
& \text { ) ; }
\end{aligned}
$$


Note that $X_{5}$ and $X_{10}$ are fixed to 1 to identify the scale of the two factors, and the lower bounds of $X_{17}$ and $X_{19}$ are set to 0 to ensure that the factor variances are nonnegative. Technically, choosing a model identification method is an arbitrary decision, and it should not affect the final results. Usually, practical

$$
\begin{aligned}
& F=\left[\begin{array}{cc}
.813 & .016 \\
-.020 & .813
\end{array}\right] \quad \Psi=\left[\begin{array}{cc}
.36 & .209 \\
.209 & .36
\end{array}\right] \\
& {\left[\begin{array}{ll}
1.004 & 0 \\
1.008 & 0
\end{array}\right] \quad\left[\begin{array}{ll}
.111 & \\
& .093
\end{array}\right.} \\
& H=\left[\begin{array}{cc}
1.008 & 0 \\
.999 & 0 \\
0 & .983 \\
0 & 1.004 \\
0 & 1.001
\end{array}\right] \quad \Theta=[
\end{aligned}
$$

concerns, such as programming convenience and/or personal preference, can play a role in making the decision.

After submitting the program in Listing 2, the SAS output window shows the ML estimates in matrix notation. As expected, the parameters are recovered very well.
In summary, a user needs to provide the required information (i.e., the data set to be analyzed, the variables, model specification, starting values, and the optimization options) to those \%LET statements, and the SAS/IML program will be called to fit the SSM.

\section{A simulation experiment}

In order to better evaluate the performance of the SAS/IML program, we replicated the simulation design in Zhang et al. (2008). ${ }^{4}$ Two conditions were fully crossed and replicated 100 times in this experiment. First, the length of the series had three levels $(T=50,100$, and 200). Second, the amount of the measurement error had four levels. That is, $\Theta_{1}=\operatorname{diag}[.1, .1, .1$, $.1, .1, .1], \Theta_{2}=\operatorname{diag}[.3, .3, .3, .3, .3, .3], \Theta_{3}=\operatorname{diag}[.5, .5, .5, .5$, $.5, .5]$, and $\Theta_{4}=\operatorname{diag}[1,1,1,1,1,1]$. Table 1 shows the results, and the parameter elements are organized in the same order as in the table in Zhang et al. (2008, p. 393).

The averaged ML estimates in Table 1 show that most parameter elements are recovered satisfactorily. However, notable bias can be observed for some of the parameter elements. Particularly, the factor loadings are underestimated by about $10 \%$ when the length of the series is 50 and the amount of measurement error is 1 , indicating that the length of time series in this model may not be sufficient, and a longer time series is needed for the model considered. In general, the length of 100 is the rule of thumb in econometrics and time series analysis.

\footnotetext{
${ }^{4}$ The SAS/IML program illustrated in Listing 1 is customized into a SAS macro program for this simulation experiment. All SAS programs used in this article can be downloaded from www.cete.us/research/publications.
}

The standard deviations of the ML estimates across replications are provided in Table 2. In general, the magnitude of the standard deviations is reasonable, reflecting the stability of the estimates. The pattern of the magnitude shows that the amount of measurement error tends to decrease the stability of the estimates, whereas the length of the time series tends to increase the stability.

As compared to the results reported by Zhang et al. (2008, p. 393) using MKFM6, the results in this experiment are close, although the data analyzed were not exactly the same in the two simulations. Yet this SAS/IML program encountered fewer numeric problems in the simulation. Specifically, 50 models in Zhang et al. (2008) were excluded from their results, whereas only 13 models were excluded from this simulation experiment, in which 11 models did not reach convergence (six models in which length $=50$ and measurement error $=0.1$, and five models in which length $=$ 100 and measurement error $=0.1)$ and two models produced exceedingly large estimates for $X_{3}$ (35.30 in one case and 43.54 in the other).

\section{Discussion}

This article introduces a SAS/IML program for estimating the parameters of a general linear model in state space form. We showed that the parameter estimation was tested with satisfaction in a simulation experiment. The IML code is open to all users and can be tailored to other purposes. In this section, some remaining issues and limitations are discussed.

First, unlike MKFM6, the current version of this program does not provide asymptotic standard error $(S E)$ estimates. 
Table 1 Maximum-likelihood estimates from the SAS/IML program using the Kalman filter algorithm

\begin{tabular}{|c|c|c|c|c|c|c|c|c|c|c|c|c|c|}
\hline \multirow{4}{*}{$\begin{array}{l}\text { Labels of Model } \\
\text { Parameters }\end{array}$} & \multirow{4}{*}{$\begin{array}{l}\text { True Population } \\
\text { Parameter Values }\end{array}$} & \multicolumn{12}{|c|}{ Amount of Measurement Error } \\
\hline & & \multicolumn{3}{|l|}{0.1} & \multicolumn{3}{|l|}{0.3} & \multicolumn{3}{|l|}{0.5} & \multicolumn{3}{|l|}{1} \\
\hline & & \multicolumn{3}{|c|}{ Time Series Length } & \multicolumn{3}{|c|}{ Time Series Length } & \multicolumn{3}{|c|}{ Time Series Length } & \multicolumn{3}{|c|}{ Time Series Length } \\
\hline & & $50^{\mathrm{a}}$ & $100^{\mathrm{b}}$ & $200^{\mathrm{c}}$ & $50^{\mathrm{c}}$ & $100^{\mathrm{c}}$ & $200^{\mathrm{c}}$ & $50^{\mathrm{c}}$ & $100^{\mathrm{c}}$ & $200^{\mathrm{c}}$ & $50^{\mathrm{d}}$ & $100^{\mathrm{c}}$ & $200^{\mathrm{c}}$ \\
\hline$X_{5}\left(\lambda_{11}\right)$ & 1 & 0.97 & 0.99 & 0.98 & 0.93 & 0.97 & 0.99 & 0.96 & 0.98 & 0.99 & 0.89 & 0.94 & 0.99 \\
\hline$X_{7}\left(\lambda_{21}\right)$ & 1 & 0.97 & 0.99 & 0.98 & 0.92 & 0.97 & 0.98 & 0.94 & 0.97 & 0.99 & 0.91 & 0.97 & 0.98 \\
\hline$X_{9}\left(\lambda_{31}\right)$ & 1 & 0.97 & 0.99 & 0.98 & 0.92 & 0.97 & 0.97 & 0.96 & 0.98 & 1.00 & 0.90 & 0.96 & 0.98 \\
\hline$X_{12}\left(\lambda_{42}\right)$ & 1 & 0.95 & 0.99 & 0.99 & 0.93 & 1.00 & 1.01 & 0.94 & 0.97 & 0.98 & 0.89 & 0.95 & 0.98 \\
\hline$X_{14}\left(\lambda_{52}\right)$ & 1 & 0.94 & 0.99 & 0.99 & 0.95 & 0.99 & 1.01 & 0.94 & 0.96 & 0.98 & 0.86 & 0.95 & 0.99 \\
\hline$X_{16}\left(\lambda_{62}\right)$ & 1 & 0.94 & 0.98 & 0.99 & 0.95 & 0.98 & 1.01 & 0.94 & 0.97 & 0.99 & 0.88 & 0.96 & 0.99 \\
\hline$X_{20}\left(q_{1}\right)$ & & 0.10 & 0.10 & 0.10 & 0.28 & 0.29 & 0.29 & 0.51 & 0.48 & 0.51 & 1.01 & 0.97 & 0.99 \\
\hline$X_{21}\left(q_{2}\right)$ & & 0.09 & 0.10 & 0.10 & 0.32 & 0.30 & 0.29 & 0.48 & 0.50 & 0.50 & 0.99 & 1.00 & 0.99 \\
\hline$X_{22}\left(q_{3}\right)$ & & 0.10 & 0.10 & 0.10 & 0.30 & 0.29 & 0.30 & 0.46 & 0.50 & 0.49 & 0.98 & 0.99 & 1.01 \\
\hline$X_{23}\left(q_{4}\right)$ & & 0.10 & 0.10 & 0.10 & 0.30 & 0.30 & 0.30 & 0.49 & 0.49 & 0.50 & 0.96 & 0.99 & 0.98 \\
\hline$X_{24}\left(q_{5}\right)$ & & 0.10 & 0.10 & 0.10 & 0.29 & 0.29 & 0.29 & 0.50 & 0.50 & 0.50 & 0.98 & 0.98 & 0.99 \\
\hline$X_{25}\left(q_{6}\right)$ & & 0.09 & 0.10 & 0.10 & 0.29 & 0.30 & 0.30 & 0.51 & 0.50 & 0.50 & 0.94 & 0.95 & 1.00 \\
\hline$X_{1}\left(b_{11}\right)$ & 0.8 & 0.77 & 0.78 & 0.79 & 0.78 & 0.78 & 0.79 & 0.73 & 0.77 & 0.78 & 0.76 & 0.79 & 0.79 \\
\hline$X_{2}\left(b_{12}\right)$ & 0 & -0.01 & 0.00 & 0.00 & -0.02 & 0.00 & 0.01 & 0.00 & 0.00 & 0.00 & 0.00 & -0.01 & 0.00 \\
\hline$X_{3}\left(b_{21}\right)$ & 0 & 0.03 & 0.00 & 0.00 & 0.01 & 0.00 & 0.00 & 0.00 & 0.00 & 0.00 & 0.03 & 0.00 & 0.00 \\
\hline$X_{4}\left(b_{22}\right)$ & 0.8 & 0.73 & 0.77 & 0.79 & 0.72 & 0.77 & 0.79 & 0.74 & 0.77 & 0.79 & 0.73 & 0.77 & 0.79 \\
\hline$X_{18}\left(d_{12}\right)$ & 0.18 & 0.18 & 0.18 & 0.18 & 0.17 & 0.18 & 0.18 & 0.17 & 0.19 & 0.18 & 0.19 & 0.18 & 0.18 \\
\hline
\end{tabular}

${ }^{\mathrm{a}}$ Based on 94 replications. ${ }^{\mathrm{b}}$ Based on 95 replications. ${ }^{\mathrm{c}}$ Based on 100 replications. ${ }^{\mathrm{d}}$ Based on 98 replications. The labels $(\lambda \mathrm{s}, q \mathrm{~s}, b \mathrm{~s}$, and $d)$ used in Zhang et al. (2008) are included in parentheses for reference.

The standard deviations provided in Table 2 will converge to the true standard errors as the number of simulation replications goes to infinity. For a given sample, one alternative solution to use in getting $S E$ s is a bootstrap method. For time series data, however, the standard nonparametric bootstrap (i.e., simple random sampling with replacement) is not appropriate, because it destroys the lead-lag relationships in time, and consequently will give misleading $S E$ estimates. The bootstrap methods appropriate for time series include the parametric bootstrap (also known as a "Monte Carlo resampling method") and the residual-based bootstrap (Stoffer \& Wall, 1991, 2004). A parametric bootstrap is essentially a Monte Carlo simulation in which the population parameters are estimates from the original sample and repeated bootstrap samples are simulated on the basis of the data-generating mechanism implied by the specified model. The underlying assumptions of parametric bootstrap are that the specified model is correct in the population and that the time series data conform to a certain distribution. The most commonly used distribution is a (multivariate) normal distribution.

The residual-based bootstrap is considered a semiparametric approach because, on the one hand, the population parameters are taken to be sample estimates assuming that the specified model is correct, which is the same as in a parametric bootstrap; on the other hand, random samples are drawn, with replacement, from the standardized residuals, as in the standard nonparametric bootstrap. The basic idea behind the residual-based bootstrap is that the standardized residuals are independent after all dynamic and measurement relationships have been accounted for by the model. Taking the bootstrapped standardized residuals and the observations reconstructed via the specified model, a bootstrap sample can be obtained. Stoffer and Wall (1991) showed that bootstrap $S E$ s are asymptotically consistent and are of definite value over the conventional asymptotic $S E$ s. This procedure, however, is not robust against model misspecification (Stoffer \& Wall, 1991; Zhang \& Chow, 2010).

With either bootstrap method, bootstrap samples are constructed, and the same model is fit to the bootstrap samples to obtain point estimates. Finally, $S E$ s can be calculated as standard deviations from the retained point estimates across bootstrap replications.

Second, some literatures for SSM (particularly in econometrics) often include fixed regressors in the model 
Table 2 Standard deviations of maximum-likelihood estimates across replications

\begin{tabular}{|c|c|c|c|c|c|c|c|c|c|c|c|c|}
\hline \multirow{4}{*}{$\begin{array}{l}\text { Labels of Model } \\
\text { Parameters }\end{array}$} & \multicolumn{12}{|c|}{ Amount of Measurement Error } \\
\hline & \multicolumn{3}{|l|}{0.1} & \multicolumn{3}{|l|}{0.3} & \multicolumn{3}{|l|}{0.5} & \multicolumn{3}{|l|}{1} \\
\hline & \multicolumn{3}{|c|}{ Time Series Length } & \multicolumn{3}{|c|}{ Time Series Length } & \multicolumn{3}{|c|}{ Time Series Length } & \multicolumn{3}{|c|}{ Time Series Length } \\
\hline & $50^{\mathrm{a}}$ & $100^{\mathrm{b}}$ & $200^{\mathrm{c}}$ & $50^{\mathrm{c}}$ & $100^{\mathrm{c}}$ & $200^{\mathrm{c}}$ & $50^{\mathrm{c}}$ & $100^{\mathrm{c}}$ & $200^{c}$ & $50^{\mathrm{d}}$ & $100^{\mathrm{c}}$ & $200^{\mathrm{c}}$ \\
\hline$X_{5}\left(\lambda_{11}\right)$ & 0.11 & 0.09 & 0.06 & 0.17 & 0.12 & 0.08 & 0.19 & 0.15 & 0.10 & 0.31 & 0.20 & 0.12 \\
\hline$X_{7}\left(\lambda_{21}\right)$ & 0.12 & 0.09 & 0.06 & 0.14 & 0.13 & 0.08 & 0.21 & 0.14 & 0.09 & 0.29 & 0.19 & 0.11 \\
\hline$X_{9}\left(\lambda_{31}\right)$ & 0.12 & 0.08 & 0.06 & 0.16 & 0.13 & 0.09 & 0.21 & 0.13 & 0.10 & 0.29 & 0.20 & 0.12 \\
\hline$X_{12}\left(\lambda_{42}\right)$ & 0.11 & 0.08 & 0.06 & 0.18 & 0.12 & 0.08 & 0.18 & 0.15 & 0.09 & 0.31 & 0.18 & 0.12 \\
\hline$X_{14}\left(\lambda_{52}\right)$ & 0.11 & 0.08 & 0.05 & 0.18 & 0.12 & 0.08 & 0.20 & 0.15 & 0.09 & 0.29 & 0.21 & 0.14 \\
\hline$X_{16}\left(\lambda_{62}\right)$ & 0.12 & 0.07 & 0.06 & 0.17 & 0.11 & 0.08 & 0.22 & 0.17 & 0.10 & 0.30 & 0.20 & 0.12 \\
\hline$X_{20}\left(q_{1}\right)$ & 0.03 & 0.02 & 0.01 & 0.08 & 0.06 & 0.05 & 0.14 & 0.08 & 0.06 & 0.31 & 0.17 & 0.14 \\
\hline$X_{21}\left(q_{2}\right)$ & 0.03 & 0.02 & 0.02 & 0.08 & 0.05 & 0.04 & 0.14 & 0.08 & 0.06 & 0.28 & 0.16 & 0.11 \\
\hline$X_{22}\left(q_{3}\right)$ & 0.03 & 0.02 & 0.02 & 0.08 & 0.05 & 0.04 & 0.13 & 0.09 & 0.06 & 0.27 & 0.17 & 0.12 \\
\hline$X_{23}\left(q_{4}\right)$ & 0.03 & 0.02 & 0.01 & 0.08 & 0.05 & 0.04 & 0.15 & 0.09 & 0.07 & 0.25 & 0.19 & 0.12 \\
\hline$X_{24}\left(q_{5}\right)$ & 0.03 & 0.02 & 0.01 & 0.09 & 0.06 & 0.04 & 0.14 & 0.09 & 0.06 & 0.26 & 0.19 & 0.12 \\
\hline$X_{25}\left(q_{6}\right)$ & 0.03 & 0.02 & 0.02 & 0.08 & 0.06 & 0.04 & 0.14 & 0.10 & 0.06 & 0.26 & 0.17 & 0.13 \\
\hline$X_{1}\left(b_{11}\right)$ & 0.12 & 0.09 & 0.05 & 0.11 & 0.10 & 0.06 & 0.19 & 0.11 & 0.08 & 0.19 & 0.12 & 0.07 \\
\hline$X_{2}\left(b_{12}\right)$ & 0.13 & 0.08 & 0.05 & 0.14 & 0.09 & 0.06 & 0.19 & 0.09 & 0.08 & 0.22 & 0.13 & 0.08 \\
\hline$X_{3}\left(b_{21}\right)$ & 0.14 & 0.09 & 0.05 & 0.13 & 0.10 & 0.06 & 0.21 & 0.10 & 0.06 & 0.27 & 0.12 & 0.07 \\
\hline$X_{4}\left(b_{22}\right)$ & 0.15 & 0.08 & 0.05 & 0.16 & 0.10 & 0.05 & 0.17 & 0.09 & 0.06 & 0.20 & 0.13 & 0.07 \\
\hline$X_{18}\left(d_{12}\right)$ & 0.04 & 0.03 & 0.02 & 0.07 & 0.05 & 0.03 & 0.08 & 0.05 & 0.03 & 0.11 & 0.07 & 0.05 \\
\hline
\end{tabular}

a Based on 94 replications. ${ }^{\mathrm{b}}$ Based on 95 replications. ${ }^{\mathrm{c}}$ Based on 100 replications. ${ }^{\mathrm{d}}$ Based on 98 replications. The labels $(\lambda \mathrm{s}, q \mathrm{~s}, b \mathrm{~s}$, and $d)$ used in Zhang et al. (2008) are included in parentheses for reference.

formulation. That is, a vector of exogenous variables can be added to the measurement equation and/or the transition equation in order to accommodate any intervention or treatment effect exerted to the subject. This feature is available in MKFM6 but is not currently implemented in the KALCVF subroutine. For advanced users, however, adding this feature in the program is feasible by writing a separate module to replace the KALCVF subroutine, if necessary.

Third, modeling multiple subjects simultaneously in a group-based SSM is a possible extension for the current program. This modification is relatively easy to accomplish in the op fun module. Specifically, a total PED can be created by adding up the PEDs from the different subjects. Then, the total PED can be optimized by the NLPQN subroutine to provide the ML estimates. This practice of fitting the same model to a group of subjects, however, is somewhat contradictory to the gist of the analyses of intraindividual variation, because "such pooling implies that the individuality of each subject in the population is immaterial to the statistical analysis - subjects in a homogeneous population are considered to be exchangeable like atoms in a homogeneous gas, constituting replications of each other" (Molenaar \& Ram, 2009, p. 255). Fundamentally, a trade-off takes place between generalizability and idiosyncracy. A philosophical discussion of this topic is beyond the scope of this article, and readers should refer to, for example, Molenaar (2004).

Fourth, it is worth noting that the expectation-maximization (EM) algorithm, first proposed by Dempster, Laird, and Rubin (1977), and later applied and extended by Shumway and Stoffer (1982) in time series analysis, provides an alternative way to optimize PED. Previous psychological applications of the EM algorithm can be found in Ho et al. (2006) and Song and Ferrer (2009). Song and Ferrer reported that the EM algorithm was simple and effective for dealing with highly nonlinear likelihood functions in their simulation study. However, Ho et al. (2006, p. 156) discussed that the EM algorithm can converge slowly in the latter stages of the iterative procedure and that it might be desirable to switch to another algorithm such as the quasi-Newton at this stage. Also, $S E$ estimates cannot be obtained directly from the EM algorithm, since the matrix of second partial derivatives is not computed. Viable solutions could include using either a numeric approximation technique in the neighborhood of the optimum or the bootstrap method. 


\section{References}

Aoki, M. (1987). State space modeling of time series. Berlin, Germany: Springer.

Chow, S.-M., Ho, M.-H. R., Hamaker, E. L., \& Dolan, C. V. (2010). Equivalence and differences between structural equation modeling and state-space modeling techniques. Structural Equation Modeling, 17, 303-332.

Chow, S.-M., Zu, J., Shifren, K., \& Zhang, G. (2011). Dynamic factor analysis models with time-varying parameters. Multivariate Behavioral Research, 46, 303-339.

Commandeur, J. J. F., Koopman, S. J., \& Ooms, M. (2011). Statistical software for state space methods. Journal of Statistical Software, 41, 1-18. Retrieved from www.jstatsoft.org/v41/i1/

Dempster, A. P., Laird, N. M., \& Rubin, D. B. (1977). Maximum likelihood from incomplete data via the EM algorithm. Journal of the Royal Statistical Society, Series B, XXX, 1-39.

Dolan, C. V. (2005). MKFM6: Multi-group, multi-subject stationary time series modeling based on the Kalman filter. Retrieved December 1, 2004, from http://users.fmg.uva.nl/cdoman/

Durbin, J., \& Koopman, S. J. (2001). Time series analysis by state space methods. New York, NY: Oxford University Press.

Hamaker, E. L., Dolan, C. V., \& Molenaar, P. C. M. (2005). Statistical modeling of the individual: Rational and application of multivariate stationary time series analysis. Multivariate Behavioral Research, 40, 207-233.

Hannan, E. J., \& Deistler, M. (1988). The statistical theory of linear systems. New York, NY: Wiley.

Harvey, A. C. (1989). Forecasting, structural time series models and the Kalman filter. Princeton, NJ: Princeton University Press.

Ho, M.-H. R., Shumway, R., \& Ombao, H. (2006). State-space approach to modeling dynamic processes: Applications in biological and social sciences. In T. A. Walls \& J. L. Schafer (Eds.), Models for intensive longitudinal data (pp. 148-170). New York, NY: Oxford University Press.

Kalman, R. E. (1960). A new approach to linear filtering and prediction problems. Transactions of the ASME-Journal of Basic Engineering (Series D), 82, 35-45.

Lütkepohl, H. (2005). New introduction to multiple time series analysis. Berlin, Germany: Springer.

Molenaar, P. C. M. (2004). A manifesto on psychology as idiographic science: Bring the person back into scientific psychology, this time forever. Measurement, 2, 201-218.
Molenaar, P. C. M., \& Nesselroade, J. R. (2009). The recoverability of P-technique factor analysis. Multivariate Behavioral Research, 44, 130-141.

Molenaar, P. C. M., \& Ram, N. (2009). Advances in dynamic factor analysis of psychological processes. In J. Valsiner, P. C. M. Molenaar, M. C. D. P. Lyra, \& N. Chaudhary (Eds.), Dynamic process methodology in the social and developmental sciences (pp. 255-268). Dordrecht, The Netherlands: Springer Science.

SAS Institute Inc. (2010). SAS/IML ${ }^{\circledR} 9.22$ user's guide. Cary, NC: SAS Institute Inc.

Schweppe, F. (1965). Evaluation of likelihood functions for Gaussian signals. IEEE Transactions on Information Theory, 11, 61-70.

Selukar, R. (2011). State space modeling using SAS. Journal of Statistical Software, 41, 1-13. Retrieved from www.jstatsoft.org/v41/i12/

Shumway, R. H., \& Stoffer, D. S. (1982). An approach to time series smoothing and forecasting using the EM algorithm. Journal of Time Series Analysis, 3, 253-264.

Song, H., \& Ferrer, E. (2009). State-space modeling of dynamic psychological processes via the Kalman smoother algorithm: Rationale, finite sample properties, and applications. Structural Equation Modeling, 16, 338-363.

Stoffer, D. S., \& Wall, K. D. (1991). Bootstrapping state-space models: Gaussian maximum likelihood estimation and the Kalman filter. Journal of the American Statistical Association, 86, 1024-1033.

Stoffer, D. S., \& Wall, K. D. (2004). Resampling in state spaace models. In A. Harvey, S. J. Koopman, \& N. Shephar (Eds.), State space and unobserved component models: Theory and applications. (pp. 171202). New York, NY: Cambridge University Press.

van Rijn, P. (2008). Categorical time series in psychological measurement (Unpublished doctoral dissertation). Amsterdam, The Netherlands: University of Amsterdam.

Wei, W. W. S. (1990). Time series analysis: Univariate and multivariate methods. Redwood City, CA: Addison-Wesley.

Zhang, G., \& Chow, S.-M. (2010). Standard error estimation in stationary multivariate time series models using residual-based bootstrap procedures. In P. C. M. Molenaar \& K. M. Newell (Eds.), Individual pathway of change: Statistical models for analyzing learning and development (pp. 169-182). Washington, DC: American Psychological Association.

Zhang, Z., Hamaker, E. L., \& Nesselroade, J. R. (2008). Comparisons of four methods for estimating a dynamic factor model. Structural Equation Modeling, 15, 377-402. 